\title{
THE PSEUDOSPECTRAL METHOD FOR THIRD-ORDER DIFFERENTIAL EQUATIONS*
}

\author{
WEIZHANG HUANG $\dagger \neq$ AND DAVID M. SLOAN $\dagger$
}

\begin{abstract}
Generalized quadrature rules are derived which assist in the selection of collocation points for the pseudospectral solution of differential equations. In particular, it is shown that for an $n$ th-order differential equation in one space dimension with two-point derivative boundary conditions, an ideal choice of interior collocation points is the set of zeros of a Jacobi polynomial. The pseudospectral solution of a third-order initial-boundary value problem is considered and accuracy is assessed by examining how well the discrete eigenproblem approximates the continuous one. Convergence is established for a special choice of collocation points and numerical results are included to demonstrate the viability of the approach.
\end{abstract}

Key words. pseudospectral method, third-order differential equation, generalized quadrature rules, Jacobi polynomials

AMS(MOS) subject classifications. 65D05, 65D32, 65N35

1. Introduction. Pseudospectral methods provide a useful alternative to finite difference and finite element methods for the approximate solution of differential equations. Theoretical studies and numerical experience have confirmed that for problems with smooth solutions pseudospectral methods converge much faster than finite difference or finite element methods [2]. Furthermore, nonlinearities do not create any special difficulties. Pseudospectral methods have gained popularity in recent years and they now feature in a wide range of applications [1].

In the pseudospectral method each dependent variable in the differential problem is approximated by a polynomial of finite degree. The discrete approximating equations are then obtained by setting residuals to zero at an appropriate set of collocation points in the solution domain. The proper choice of collocation points is crucial in terms of accuracy, stability, and ease of implementation of boundary conditions. A set of collocation points is typically chosen as the set of nodes in an appropriate Gauss quadrature formula. For equations of order 1 or 2 it is known that collocation points are the Gauss-Radau or Gauss-Lobatto nodes, respectively [2]. Interesting work by Malek and Phillips [7] has recently shown that for fourth-order problems the ideal choice of interior collocation points is the set of zeros of certain Gegenbauer polynomials, these being nodes for some generalized Gaussian quadrature rules. Generalizations of Gaussian quadrature rules are discussed, for example, by Golub and Kautsky [4].

The objective of this paper is to construct generalized Gaussian quadrature rules which assist in the choice of interior collocation points for the pseudospectral approximation of model differential equations of any finite order. The rules are then used to construct pseudospectral methods for third-order boundary value or initial-boundary value problems. The third-order problem is of mathematical interest since it lacks the symmetry of the second-order problem [11]. It is of physical interest since it contains the type of operator which appears in many commonly-occurring partial differential equations such as the Korteweg-de Vries equation. Pseudospectral methods for a model third-order problem have been considered by Mulholland [8].

* Received by the editors August 7, 1991; accepted for publication (in revised form) January 5, 1992.

$\uparrow$ Department of Mathematics, University of Strathclyde, Glasgow G1 1XH, United Kingdom.

$\$$ Institute of Applied Mathematics, Academia Sinica, Beijing 100080, People's Republic of China. The research of this author was supported by a Royal Fellowship Award from the Royal Society of London. 
In $\S 2$ of the paper we consider generalized quadrature rules. A function interpolation approach is used, with function values specified at interior collocation points and derivative values specified at endpoints. Section 3 deals with a pseudospectral method for a third-order initial-boundary value problem. Accuracy is examined by considering how well the discrete system approximates the associated third-order differential eigenproblem. Section 4 contains numerical results on the pseudospectral solution of some model third-order boundary value problems and the final section contains comments.

2. Generalized quadrature rules. Generalized quadrature rules play an important role in theory and applications of pseudospectral methods. In this section we will follow the approach of Malek and Phillips [7] to construct a generalized quadrature rule which helps us to choose the collocation points of the pseudospectral approximation to third-order differential equations.

Quadrature formulas are often constructed from interpolating polynomials. In this way we can, in many cases, obtain quadrature formulas that are convenient to use and that will give sufficiently accurate results. Therefore, at the beginning of this section we consider a function interpolation problem.

2.1. Interpolation with multiple nodes. We assume that we are given $N$ (maybe $N-1)^{1}$ distinct nodes $-1=x_{1}<x_{2}<\cdots<x_{N-1}<x_{N}=1$ in the segment $[-1,1]$ and that at these nodes we are given the values of the function $f(x)$ and its derivatives as follows:

$$
\begin{aligned}
f_{k} & =f\left(x_{k}\right), & & 2 \leqq k \leqq N-1, \\
f_{1}^{(\nu)} & =f^{(\nu)}\left(x_{1}\right), & & 0 \leqq \nu \leqq l_{n}, \\
f_{N}^{(\nu)} & =f^{(\nu)}\left(x_{N}\right), & & 0 \leqq \nu \leqq r_{n},
\end{aligned}
$$

where integers $l_{n}$ and $r_{n}$ are given such that

$$
l_{n}+r_{n}+2=n, \quad l_{n} \geqq-1, \quad r_{n} \geqq-1,
$$

and $n$ is a natural number.

It is known (see, for example, [6, pp. 45-49]) that the polynomial $P_{N+n-3}$ of degree $N+n-3$ interpolating data (2.1) is given by

$$
P_{N+n-3}(x)=\sum_{k=2}^{N-1} f_{k} h_{k}(x)+\sum_{\nu=0}^{l_{n}} f_{1}^{(\nu)} h_{1}^{(\nu)}(x)+\sum_{\nu=0}^{r_{n}} f_{N}^{(\nu)} h_{N}^{(\nu)}(x)
$$

where

$$
\begin{aligned}
h_{k}(x) & =\frac{\pi(x)}{\pi^{\prime}\left(x_{k}\right)\left(x-x_{k}\right)} \cdot \frac{(1+x)^{l_{n}+1}}{\left(1+x_{k}\right)^{l_{n}+1}} \cdot \frac{(x-1)^{r_{n}+1}}{\left(x_{k}-1\right)^{r_{n}+1}}, & & 2 \leqq k \leqq N-1, \\
h_{1}^{(\nu)}(x) & =S_{1}(x) \sum_{i=\nu}^{l_{n}} a_{i}^{(\nu)}(x+1)^{i}, & & 0 \leqq \nu \leqq l_{n}, \\
h_{N}^{(\nu)}(x) & =S_{N}(x) \sum_{i=\nu}^{r_{n}} b_{i}^{(\nu)}(x-1)^{i}, & & 0 \leqq \nu \leqq r_{n},
\end{aligned}
$$

${ }^{1}$ From (2.1) it can be seen that if $l_{n}=-1$ only $N-1$ nodes $x_{2}<x_{3}<\cdots<x_{N-1}<x_{N}=1$ are used in the segment $(-1,1]$ and if $r_{n}=-1$ only $N-1$ nodes $-1=x_{1}<x_{2}<\cdots<x_{N-1}$ are used in $[-1,1)$. However, to simplify the presentation we shall henceforth refer to $N$ nodes. 
and

$$
\begin{aligned}
\pi(x) & =\prod_{i=2}^{N-1}\left(x-x_{i}\right), \\
S_{1}(x) & =\frac{\pi(x)}{\pi\left(x_{1}\right)} \cdot \frac{(x-1)^{r_{n}+1}}{\left(x_{1}-1\right)^{r_{n}+1}}, \\
S_{N}(x) & =\frac{\pi(x)}{\pi\left(x_{N}\right)} \cdot \frac{(x+1)^{l_{n}+1}}{\left(x_{N}+1\right)^{l_{n}+1}}, \\
a_{\nu}^{(\nu)} & =\frac{1}{\nu !}, \quad a_{i}^{(\nu)}=-\sum_{j=\nu}^{i-1} \frac{a_{j}^{(\nu)}}{(i-j) !} S_{1}^{(i-j)}\left(x_{1}\right), \quad \nu+1 \leqq i \leqq l_{n}, \quad 0 \leqq \nu \leqq l_{n}, \\
b_{\nu}^{(\nu)} & =\frac{1}{\nu !}, \quad b_{i}^{(\nu)}=-\sum_{j=\nu}^{i-1} \frac{b_{j}^{(\nu)}}{(i-j) !} S_{N}^{(i-j)}\left(x_{N}\right), \quad \nu+1 \leqq i \leqq r_{n}, \quad 0 \leqq \nu \leqq r_{n} .
\end{aligned}
$$

For the remainder of this section on interpolation we have the following lemma.

LEMMA 2.1. If $f(x)$ has a continuous derivative of order $N+n-2$ on $[-1,1]$ then the remainder of $(2.3)$ is

$$
\begin{aligned}
R_{N}[f ; x] & \equiv f(x)-P_{N+n-3}(x) \\
& =\frac{f^{(N+n-2)}\left(\xi_{x}\right)}{(N+n-2) !} \pi(x)(x+1)^{l_{n}+1}(x-1)^{r_{n}+1},
\end{aligned}
$$

where $-1<\xi_{x}<1$ for $x \in[-1,1]$.

2.2. Quadrature formulas. By using the interpolating polynomial (2.3) we can construct a quadrature formula as follows:

$$
\int_{-1}^{1} \omega_{\alpha, \beta}(x) f(x) d x=\sum_{k=2}^{N-1} f_{k} \omega_{k}+\sum_{\nu=0}^{l_{n}} \omega_{1}^{(\nu)} f_{1}^{(\nu)}+\sum_{\nu=0}^{r_{n}} \omega_{N}^{(\nu)} f_{N}^{(\nu)}+E_{N}[f],
$$

where the weights are given by

$$
\begin{aligned}
\omega_{k} & =\int_{-1}^{1} \omega_{\alpha, \beta}(x) h_{k}(x) d x, & & 2 \leqq k \leqq N-1, \\
\omega_{1}^{(\nu)} & =\int_{-1}^{1} \omega_{\alpha, \beta}(x) h_{1}^{(\nu)}(x) d x, & & 0 \leqq \nu \leqq l_{n}, \\
\omega_{N}^{(\nu)} & =\int_{-1}^{1} \omega_{\alpha, \beta}(x) h_{N}^{(\nu)}(x) d x, & & 0 \leqq \nu \leqq r_{n},
\end{aligned}
$$

and $\omega_{\alpha, \beta}(x)$ is a weight function, which is defined by

$$
\omega_{\alpha, \beta}(x)=(1-x)^{\alpha}(1+x)^{\beta}, \quad-2-r_{n}<\alpha, \quad-2-l_{n}<\beta .
$$

The term $E_{N}[f]$ denotes the truncation error of the quadrature formula.

We notice that the integral on the left-hand side of (2.7) may not exist for some function. Thus, we have to restrict our discussion to the function space

$$
B_{\alpha, \beta}=\left\{f: f \text { is measurable and } \int_{-1}^{1} \omega_{\alpha . \beta}(x) f(x) d x<+\infty\right\} .
$$

Before we discuss the algebraic degree of precision of (2.7), we give a result about the existence of integral $\int_{-1}^{1} \omega_{\alpha, \beta}(x) f(x) d x$, which is useful for our problem. 
LEMMA 2.2. Suppose that the function $u(x)$ on $[-1,1]$ satisfies the boundary conditions

$$
\begin{array}{ll}
u^{(\nu)}(-1)=0, & 0 \leqq \nu \leqq l_{n}, \\
u^{(\nu)}(+1)=0, & 0 \leqq \nu \leqq r_{n},
\end{array}
$$

where $r_{n}, l_{n}$ are defined by (2.2), and suppose that $u(x)$ has a continuous derivative of order

$$
r \geqq q \quad\left(\equiv \max \left(r_{n}+1, l_{n}+1\right)\right) .
$$

Then (i)

$$
\tilde{u}(x)=\frac{u(x)}{(1-x)^{r_{n}+1}(1+x)^{l_{n}+1}}
$$

is well defined;

$$
\int_{-1}^{1} \omega_{\alpha+r_{n}+1, \beta+l_{n}+1}(x)|\tilde{u}(x)| d x<+\infty
$$

implies

$$
\int_{-1}^{1} \omega_{\alpha, \beta}(x)|u(x)| d x<+\infty
$$

(iii) If $u^{(p)}(x), q \leqq p \leqq r$, exist, then $\tilde{u}^{(p)}, 0 \leqq p \leqq r-q$, also exist.

Proof. It is not difficult to obtain (i)-(iii) by using the assumptions and the Taylor expansion formula with Cauchy remainder.

From (2.9) and this lemma it is obvious that any bounded function $u(x)$ on $[-1,1]$ belongs to $B_{\alpha, \beta}$ when $\alpha>-1$ and $\beta>-1$. Furthermore, any function $u(x)$ that satisfies (2.11) and has a bounded $\tilde{u}(x)$ defined by (2.12), belongs to $B_{\alpha, \beta}$ when $\alpha>-2-r_{n}$ and $\beta>-2-l_{n}$. Thus, $\mathbf{P}_{m}$, which denotes the space containing all polynomials on $[-1,1]$ of degree not greater than $m$, is contained in $B_{\alpha, \beta}$ when $\alpha>-1$ and $\beta>-1$, and all polynomials satisfying (2.11) belong to $B_{\alpha, \beta}$ when $\alpha>-2-r_{n}$ and $\beta>-2-l_{n}$. Also see Lemma 3.4 and Note 3.2.

Now let us consider the algebraic degree of precision and the remainder of quadrature formula (2.7).

From Lemma 2.1 the following lemma is trivial.

LEMмA 2.3. The quadrature rule (2.7) is exact for all polynomials $p(x) \in$ $\mathbf{P}_{N+n-3} \cap B_{\alpha, \beta}$.

Then we have Theorem 2.1.

THEOREM 2.1. Suppose that the interior nodes $x_{k}, 2 \leqq k \leqq N-1$, are chosen to be the zeros of the Jacobi polynomial $P_{N-2^{n}}^{\left(\alpha+r_{n}+\beta+l_{n}+1\right)}(x)$ of degree $N-2$. Then the quadrature formula (2.7) is exact for all

$$
p(x) \in \mathbf{P}_{2 N+n-5} \cap B_{\alpha, \beta} .
$$

Proof. Any polynomial $p(x) \in \mathbf{P}_{2 N+n-5} \cap B_{\alpha, \beta}$ can be written as

$$
\begin{aligned}
p(x)= & (x-1)^{r_{n}+1}(x+1)^{l_{n}+1} P_{N-2^{n}}^{\left(\alpha+r_{n}+\beta+l_{n}+1\right)}(x) \\
& \cdot Q_{N-3}(x)+R_{N+n-3}(x),
\end{aligned}
$$


where $Q_{N-3}(x) \in \mathbf{P}_{N-3}$ and $R_{N+n-3} \in \mathbf{P}_{N+n-3} \cap B_{\alpha, \beta}$ are the quotient and remainder, respectively, when $p(x)$ is divided by $(x-1)^{r_{n}+1}(x+1)^{l_{n}+1} P_{N-2^{n}}^{\left(\alpha+r_{n}+1, \beta+l_{n}+1\right)}(x)$. Upon multiplying $(2.13)$ by $\omega_{\alpha, \beta}(x)$ and integrating over $[-1,1]$ we obtain

$$
\begin{aligned}
\int_{-1}^{1} \omega_{\alpha, \beta}(x) p(x) d x= & \int_{-1}^{1} \omega_{\alpha+r_{n}+1, \beta+l_{n}+1}(x)(-1)^{r_{n}+1} \\
& \cdot P_{N-2^{n}}^{\left(\alpha+r_{n}+1, \beta+l_{n}+1\right)} Q_{N-3} d x \\
& +\int_{-1}^{1} \omega_{\alpha, \beta}(x) R_{N+n-3}(x) .
\end{aligned}
$$

Having noticed that $P_{N-2^{n}}^{\left(\alpha+r_{n}+1, \beta+l_{n}+1\right)}(x)$ is orthogonal to $Q_{N-3}(x)$ with respect to weight $\omega_{\alpha+r_{n}+1, \beta+l_{n}+1}(x)$ and applying Lemma 2.3 to the second term of the right-hand side of (2.14), we have

$$
\begin{aligned}
\int_{-1}^{1} \omega_{\alpha, \beta}(x) p(x) d x= & \sum_{k=2}^{N-1} \omega_{k} R_{N+n-3}\left(x_{k}\right) \\
& +\sum_{\nu=0}^{l_{n}} \omega_{1}^{(\nu)} \frac{d^{\nu} R_{N+n-3}}{d x^{\nu}}(-1) \\
& +\sum_{\nu=0}^{r_{n}} \omega_{N}^{(\nu)} \frac{d^{\nu} R_{N+n-3}}{d x^{\nu}}(+1) .
\end{aligned}
$$

Using the assumption about $x_{k}, 2 \leqq k \leqq N-1,(2.13)$ implies that

$$
\begin{aligned}
p\left(x_{k}\right) & =R_{N+n-3}\left(x_{k}\right), & & 2 \leqq k \leqq N-1, \\
\frac{d^{\nu} p}{d x^{\nu}}(-1) & =\frac{d^{\nu} R_{N+n-3}}{d x^{\nu}}(-1), & & 0 \leqq \nu \leqq l_{n}, \\
\frac{d^{\nu} p}{d x^{\nu}}(+1) & =\frac{d^{\nu} R_{N+n-3}}{d x^{\nu}}(+1), & & 0 \leqq \nu \leqq r_{n} .
\end{aligned}
$$

Combining (2.15) with (2.16), the statement of the theorem follows.

THEOREM 2.2. Suppose that the assumption of Theorem 2.1 is satisfied and that $f(x) \in B_{\alpha, \beta}$ has a continuous derivative of order $2 N+n-4$ on $[-1,1]$. Then the truncation error of (2.7) is given by

$$
\begin{aligned}
E_{N}[f]= & \frac{(-1)^{r_{n}+1} f^{(2 N+n-4)}(\eta)}{(2 N+n-4) !} \\
& \cdot \int_{-1}^{1} \omega_{\alpha+r_{n}+1, \beta+l_{n}+1}(x)(\pi(x))^{2} d x,
\end{aligned}
$$

where $-1<\eta<1$.

Proof. Let $P_{2 N+n-5}(x)$ be the polynomial interpolating the data

$$
\begin{array}{ll}
f\left(x_{k}\right), f^{\prime}\left(x_{k}\right), & 2 \leqq k \leqq N-1, \\
f^{(\nu)}(-1), & 0 \leqq \nu \leqq l_{n}, \\
f^{(\nu)}(+1), & 0 \leqq \nu \leqq r_{n} .
\end{array}
$$


Then

$$
\begin{aligned}
\tilde{R}_{N}[f ; x] & \equiv f(x)-P_{2 N+n-5}(x) \\
& =\frac{(-1)^{r_{n}+1} f^{(2 N+n-4)}\left(\xi_{x}\right)}{(2 N+n-4) !}[\pi(x)]^{2}(1+x)^{l_{n}+1}(1-x)^{r_{n}+1},
\end{aligned}
$$

where $-1<\xi_{x}<1$ and $P_{2 N+n-5} \in B_{\alpha, \beta}$. Multiplying (2.19) by $\omega_{\alpha, \beta}(x)$ and integrating over $[-1,1]$, it follows that

$$
\begin{aligned}
\int_{-1}^{1} \omega_{\alpha, \beta}(x) f(x) d x= & \int_{-1}^{1} \omega_{\alpha, \beta}(x) P_{2 N+n-5}(x) d x \\
& +\frac{(-1)^{r_{n}+1}}{(2 N+n-4) !} \\
& \cdot \int_{-1}^{1} \omega_{\alpha, \beta}(x) f^{(2 N+n-4)}\left(\xi_{x}\right)[\pi(x)]^{2}(1+x)^{l_{n}+1}(1-x)^{r_{n}+1} d x .
\end{aligned}
$$

Then (2.17) follows by applying Theorem 2.1 and the mean-value theorem to the first term and second term of the right-hand side of the above equation, respectively, and by using the data (2.18).

2.3. The weights of the quadrature rule. In the applications of quadrature formula (2.7) the properties of the weights are often needed. In this section we will give some results about the weights under the assumption of Theorem 2.1.

First, we have the following.

THEOREM 2.3. $\omega_{k}>0,2 \leqq k \leqq N-1$.

Proof. For any $k, 2 \leqq k \leqq N-1$, define

$$
f_{k}(x)=\left[\prod_{\substack{i=2 \\ i \neq k}}^{N-1} \frac{x-x_{i}}{x_{k}-x_{i}}\right]^{2} \frac{(1+x)^{l_{n}+1}}{\left(1+x_{k}\right)^{l_{n}+1}} \cdot \frac{(1-x)^{r_{n}+1}}{\left(1-x_{k}\right)^{r_{n}+1}}
$$

It is obvious that $f_{k}(x) \in \mathbf{P}_{2 N+n-6} \cap B_{\alpha, \beta}$ and $f_{k}(x) \geqq 0, f_{k}(x) \not \equiv 0$. By substituting (2.20) in (2.7), we obtain that $\omega_{k}=\int_{-1}^{1} \omega_{\alpha, \beta}(x) f_{k}(x) d x>0$.

For the representations of the weights, noticing that

$$
\pi(x)=\frac{1}{\gamma_{N-2}} P_{N-2^{n}}^{\left(\alpha+r_{n}+1, \beta+l_{n}+1\right)}(x)
$$

where $\gamma_{N-2}$ is the leading coefficient of $P_{N-2^{n}}^{\left(\alpha+r_{n}+1, \beta+l_{n}+1\right)}$, using the properties of Jacobi polynomials and the Christoffel-Darboux formula (see Szegö [9] and Erdélyi [3]), we can establish

$$
\begin{aligned}
& a_{\nu}^{(\nu)}=\frac{1}{\nu !} \\
& a_{i}^{(\nu)}=-\frac{\left(r_{n}+1\right) !}{\left(\begin{array}{c}
N+\beta+l_{n}-1 \\
N-2
\end{array}\right)} \sum_{j=\nu}^{i-1}\left[\frac{(-1)^{i-j} a_{j}^{(\nu)}}{2^{i-j}(i-j) !} \cdot \sum_{l=0}^{\min \left\{(i-j), r_{n}+1\right\}} \frac{c_{i-j}^{l}}{\left(r_{n}+1-l\right) !}\right. \\
&\left.\cdot\left(N+\alpha+\beta+r_{n}+l_{n}+1\right)_{i-j-l}\left(\begin{array}{c}
N+\beta+l_{n}-1 \\
N-i+j+l-2
\end{array}\right)\right], \\
& \nu+1 \leqq i \leqq l_{n}, \quad 0 \leqq \nu \leqq l_{n} ;
\end{aligned}
$$




$$
\begin{aligned}
b_{\nu}^{(\nu)}=\frac{1}{\nu !} & \\
b_{i}^{(\nu)}=-\frac{\left(l_{n}+1\right) !}{\left(\begin{array}{c}
N+\alpha+r_{n}-1 \\
N-2
\end{array}\right)} \sum_{j=\nu}^{i-1}\left[\frac{b_{j}^{(\nu)}}{2^{i-j}(i-j) !} \cdot \sum_{l=0}^{\left.\min \left\{(i-j), l_{n}+1\right)\right\}} \frac{c_{i-j}^{l}}{\left(l_{n}+1-l\right) !}\right. & \left.\cdot\left(N+\alpha+\beta+r_{n}+l_{n}+1\right)_{i-j-1}\left(\begin{array}{c}
N+\alpha+r_{n}-1 \\
N-i+j+l-2
\end{array}\right)\right], \\
\nu+1 \leqq i \leqq r_{n}, \quad 0 \leqq \nu \leqq r_{n} ; & 0 \leqq
\end{aligned}
$$

and

$$
\begin{aligned}
\omega_{k}= & \frac{2^{\left(\alpha+\beta+r_{n}+l_{n}+1\right)}\left(2 N+\alpha+\beta+r_{n}+l_{n}\right)^{2}}{(N-1)^{2}\left(N+\alpha+\beta+r_{n}+l_{n}+1\right)^{2}} \frac{\Gamma\left(N+\alpha+r_{n}\right) \Gamma\left(N+\beta+l_{n}\right)}{(N-2) ! \Gamma\left(N+\alpha+\beta+r_{n}+l_{n}+1\right)} \\
& \cdot \frac{1}{\left(1+x_{k}\right)^{l_{n}\left(1-x_{k}\right)^{r_{n}}\left[P_{N-1}^{\left(\alpha+r_{n}+1, \beta+l_{n}+1\right)}\left(x_{k}\right)\right]^{2}}, \quad 2 \leqq k \leqq N-1} \\
\omega_{1}^{(\nu)}= & \frac{\Gamma\left(N+\alpha+r_{n}\right)}{(N-2) !\left(\begin{array}{c}
\left.N+\beta+l_{n}-1\right) \\
N-2
\end{array}\right)} \\
& \cdot \sum_{i=\nu}^{l_{n}} \frac{2^{\alpha+\beta+i+1} \Gamma(\beta+i+1)\left(N+l_{n}-i-2\right) !}{\left(l_{n}-i\right) ! \Gamma\left(N+\alpha+\beta+r_{n}+i+1\right)} a_{i}^{(\nu)}, \quad 0 \leqq \nu \leqq l_{n} \\
\omega_{N}^{(\nu)}= & \frac{\Gamma\left(N+\beta+l_{n}\right)}{(N-2) !\left(\begin{array}{c}
\left.N+\alpha+r_{n}-1\right) \\
N-2
\end{array}\right)} \\
& \cdot \sum_{i=\nu}^{r_{n}} \frac{(-1)^{i} 2^{\alpha+\beta+i+1} \Gamma(\alpha+i+1) \Gamma\left(N+r_{n}-i-1\right)}{\Gamma\left(r_{n}-i+1\right) \Gamma\left(N+\alpha+\beta+l_{n}+i+1\right)} b_{i}^{(\nu)}, \quad 0 \leqq \nu \leqq r_{n},
\end{aligned}
$$

where $\left(\begin{array}{c}m \\ l\end{array}\right)$ and $(m)_{l}$ are defined as $[m(m-1) \cdots(m-l+1)] / l$ ! and $m(m+1) \cdots(m+$ $l-1)$, respectively.

From (2.22) and (2.23), we get the asymptotic representations of $a_{i}^{(\nu)}$ and $b_{i}^{(\nu)}$ as follows:

$$
\begin{aligned}
a_{i}^{(\nu)}=O\left(N^{2(i-\nu)}\right), & & \nu \leqq i \leqq l_{n}, & 0 \leqq \nu \leqq l_{n}, \\
b_{i}^{(\nu)}=O\left(N^{2(i-\nu)}\right), & & \nu \leqq i \leqq r_{n}, & 0 \leqq \nu \leqq r_{n}
\end{aligned}
$$

as $N \rightarrow \infty$ for any fixed $\alpha, \beta, l_{n}$, and $r_{n}$, and therefore, from $(2.5),(2.6)$ it follows that

$$
\begin{array}{ll}
\omega_{1}^{(\nu)}=O\left(N^{-2(\nu+\beta+1)}\right), & 0 \leqq \nu \leqq l_{n}, \\
\omega_{N}^{(\nu)}=O\left(N^{-2(\nu+\alpha+1)}\right), & 0 \leqq \nu \leqq r_{n}
\end{array}
$$

as $N \rightarrow \infty$ for any fixed $\alpha, \beta, l_{n}$, and $r_{n}$. In order to construct the asymptotic formula of $\omega_{k}$, we write $(2.24)$ as

$$
\omega_{k}=\frac{2^{\left(\alpha+\beta+r_{n}+l_{n}+3\right)} \Gamma\left(N+\alpha+r_{n}\right) \Gamma\left(N+\beta+l_{n}\right)}{(N-2) ! \Gamma\left(N+\alpha+\beta+r_{n}+l_{n}+1\right)\left(1+x_{k}\right)^{l_{n}+2}\left(1-x_{k}\right)^{r_{n}+2}\left[P_{N-2^{n}}^{\left(\alpha+1, \beta+l_{n}+1\right)^{\prime}}\left(x_{k}\right)\right]^{2}}, \quad 2 \leqq k \leqq N-1 .
$$

Then, using the asymptotic properties of the first-order derivative (formula (8.8.1) in Szegö $[9$, p. 234]) and of the zeros $[9$, p. 236] of the Jacobi polynomial, we have

$$
\omega_{k}= \begin{cases}O\left((N-k)^{2 \beta+1} N^{-2(\beta+1)}\right), & 2 \leqq k \leqq[N / 2], \\ O\left((N-k)^{2 \alpha+1} N^{-2(\alpha+1)}\right), & {[N / 2] \leqq k \leqq N-1 .}\end{cases}
$$


It is interesting to notice that at nodes near the right end, $\omega_{k}=O\left(N^{-2(\alpha+1)}\right)=\omega_{N}^{(0)}$, and that at nodes near the left end, $\omega_{k}=O\left(N^{-2(\beta+1)}\right)=\omega_{1}^{(0)}$. At nodes near $x=0$, $\omega_{k}=O\left(N^{-1}\right)$. The asymptotic orders of $\omega_{k}(2 \leqq k \leqq N-1)$ and $\omega_{1}^{(0)}, \omega_{N}^{(0)}$ are independent of $n$ !

THEOREM 2.4. For any $\alpha>-r_{n}-2, \beta>-l_{n}-2$, we have

$$
\begin{aligned}
& \frac{2^{\alpha+\beta+r_{n}+l_{n}+3} \Gamma\left(\alpha+r_{n}+2\right) \Gamma\left(\beta+l_{n}+2\right)}{\Gamma\left(\alpha+\beta+r_{n}+l_{n}+4\right)} \\
& =\sum_{k=2}^{N-1} \omega_{k}\left(1-x_{k}\right)^{r_{n}+1}\left(1+x_{k}\right)^{l_{n}+1} \\
& =\sum_{2 \leqq k<[N / 2]} O\left(N^{-2\left(\beta+l_{n}+2\right)} k^{2 \beta+2 l_{n}+3}\right) \\
& \quad+\sum_{[N / 2] \leqq k \leqq N-1} O\left(N^{-2\left(\alpha+r_{n}+2\right)}(N-k)^{2 \alpha+2 r_{n}+3}\right) .
\end{aligned}
$$

Furthermore, if $\alpha>-1, \beta>-1$, then

$$
\frac{2^{\alpha+\beta+1} \Gamma(\alpha+1) \Gamma(\beta+1)}{\Gamma(\alpha+\beta+2)}=\sum_{k=2}^{N-1} \omega_{k}+O\left(N^{-\min (2(\alpha+1), 2(\beta+1))}\right) .
$$

Proof. The results are easy to prove by substituting $(1-x)^{r_{n}+1}(1+x)^{l_{n}+1}$ or 1 in (2.7) and using (2.28), (2.29), (2.31), and the asymptotic properties of the zeros of the Jacobi polynomial.

2.4. Some remarks. In previous sections we have shown that for given data (2.1) the interior nodes should be chosen to be the zeros of the Jacobi polynomial $P_{N-2^{n}}^{\left(\alpha+r_{n}+1, \beta+l_{n}+1\right)}(x)$ in order to obtain a quadrature rule with the highest algebraic degree of precision. This conclusion seems to suggest that an optimal choice for the interior collocation points is the set of zeros of $P_{N-2^{n}}^{\left(\alpha+r_{n}+1, I_{n}+1\right)}(x)$ when the pseudospectral method is applied to solve numerically $n$ th-order differential equations in one dimension subject to the boundary conditions

$$
\begin{array}{ll}
u^{(\nu)}(-1)=0, & 0 \leqq \nu \leqq l_{n}, \\
u^{(\nu)}(+1)=0, & 0 \leqq \nu \leqq r_{n} .
\end{array}
$$

\begin{tabular}{|c|c|c|c|c|c|}
\hline$n$ & $r_{n}$ & $l_{n}$ & $\begin{array}{l}\text { Interior points } \\
\text { are zeros of } \\
\quad P_{N-2}^{(\cdot, \cdot)}\end{array}$ & Examples & Name of collocation points \\
\hline 1 & $-1^{*}$ & 0 & $(\alpha, \beta+1)$ & $\begin{array}{c}\alpha=-\frac{1}{2}, \beta=-\frac{3}{2} \\
\alpha=0, \beta=-1\end{array}$ & $\begin{array}{l}\text { Gauss-Radau-Chebyshev } \\
\text { Gauss-Radau-Legendre }\end{array}$ \\
\hline 2 & 0 & 0 & $(\alpha+1, \beta+1)$ & $\begin{array}{l}\alpha=-\frac{3}{2}, \beta=-\frac{3}{2} \\
\alpha=-1, \beta=-1 \\
\alpha=-\frac{1}{2}, \beta=-\frac{1}{2}\end{array}$ & $\begin{array}{l}\text { Gauss-Lobatto-Chebyshev } \\
\text { Gauss-Lobatto-Legendre } \\
\text { Gauss-Lobatto-Chebyshev (extrema) }\end{array}$ \\
\hline 3 & 0 & 1 & $(\alpha+1, \beta+2)$ & This paper & Uadso-Loodto-cheoysnev (extrema) \\
\hline 4 & 1 & 1 & $(\alpha+2, \beta+2)$ & $\alpha=\beta=\lambda$ & See $[7]$ \\
\hline
\end{tabular}

Adopting this choice, Table 2.1 lists several examples for some known typical cases.

From the discussion in the previous subsections we know that the difference between twice the degree of interpolating polynomial (2.3) and the precision degree

TABLE 2.1

Examples of collocation points corresponding to zeros of the Jacobi polynomial $P_{N-2^{n}}^{\left(\alpha+r_{n}+\beta+l_{n}+1\right)}(x)$. $\left(\alpha+r_{n}+1>-1, \beta+l_{n}+1>-1.\right)$

* For this case, there is no boundary condition on the right end. 
of quadrature rule (2.7) is $n-1$. Therefore the difference exceeds unity when $n>2$, and it follows that the generalized quadrature rule (2.7) is more accurate than a standard Gauss quadrature rule for $n>2$.

3. Pseudospectral method for a third-order initial-boundary value model problem. Consider the pseudospectral method for the following model problem

$$
u_{t}=u_{x x x} \quad \text { in }(-1,1) \times(0, T]
$$

with initial condition

$$
u(x, 0)=u_{0}(x)
$$

and boundary conditions

$$
\begin{aligned}
u(+1, t) & =0, \\
u^{(\nu)}(-1, t) & =0, \quad 0 \leqq \nu \leqq 1 .
\end{aligned}
$$

Given $N-2$ interior collocation points $x_{k}, 2 \leqq k \leqq N-1$, the projection operation $P^{N}$ can be chosen as

$$
u^{N} \equiv P^{N} u=\sum_{k=2}^{N-1} u_{k} h_{k}(x)
$$

where $u_{k}=u\left(x_{k}\right)$ and the $h_{k}(x) \mathrm{s}$ are defined in (2.4) and (2.5) with $n=3, l_{n}=1, r_{n}=0$. Then the collocation equations are

$$
\frac{\partial u^{N}}{\partial t}\left(x_{k}, t\right)=\frac{\partial^{3} u^{N}}{\partial x^{3}}\left(x_{k}, t\right), \quad 2 \leqq k \leqq N-1
$$

with initial condition

$$
u^{N}\left(x_{k}, 0\right)=u_{0}\left(x_{k}\right), \quad 1 \leqq k \leqq N .
$$

If the $N-2$ interior collocation points are chosen as the zeros of Jacobi polynomial $P_{N-2}^{(\alpha+1, \beta+2)}$ as suggested in $\S 2$, the $h_{k}$ s can be rewritten as

$$
\begin{aligned}
h_{k}(x) & =(1-x)(1+x)^{2} \frac{P_{N-2}^{(\alpha+1, \beta+2)}(x)}{\left(x-x_{k}\right)\left(1+x_{k}\right)^{2}\left(1-x_{k}\right) P_{N-2}^{(\alpha+1, \beta+2)^{\prime}}\left(x_{k}\right)}, \\
2 & \leqq k \leqq N-1 .
\end{aligned}
$$

Throughout this section we assume that the interior collocation points are the zeros of $P_{N-2}^{(\alpha+1, \beta+2)}(x)$ and that $\alpha$ and $\beta$ assume values in $(-2, \infty)$ and $(-3, \infty)$, respectively. In the following section we shall deal specifically with the problem described by (3.1)-(3.3). We begin by giving some indication of the accuracy that might be achieved by the approximation described by (3.4)-(3.7).

3.1. The resolution of the eigenfunctions by polynomial interpolation. The analysis of resolution of the eigenfunctions by polynomial interpolation can often give a natural explanation of how well the discrete scheme (3.5) approximates to the continuous problem (3.1)-(3.3). Our analysis follows the approach of Weideman and Trefethen [11]. The starting point is the well-known Hermite formula (such as Krylov [6, p. 48]) 
for the error in polynomial interpolation (2.3):

$$
\begin{aligned}
R_{N}[f ; x] & \equiv f(x)-P_{N+n-3}(x) \\
& =\frac{A_{N+n-2}(x)}{2 \pi i} \int_{l} \frac{f(z)}{A_{N+n-2}(z)(z-x)} d z,
\end{aligned}
$$

where

$$
\begin{aligned}
A_{N+n-2}(z) & =(z-1)^{r_{n}+1}(z+1)^{l_{n}+1} \prod_{k=2}^{N-1}\left(z-x_{k}\right) \\
& =(z-1)^{r_{n}+1}(z+1)^{l_{n}+1} P_{N-2^{n}}^{\left(\alpha+r_{n}+\beta+l_{n}+1\right)}(z) / \gamma_{N-2},
\end{aligned}
$$

and $\gamma_{N-2}$ is the leading coefficient of $P_{N-2^{n}}^{\left(\alpha+r_{n}+1, \beta+l_{n}+1\right)}(x), l$ is any simple closed curve in the complex plane that encloses $[-1,1]$ in its interior. Taking absolute values yields

$$
\begin{aligned}
\left|R_{N}[f ; x]\right| \leqq & \frac{1}{2 \pi \gamma_{N-2}} \max _{-1 \leqq x \leqq 1}\left|(x-1)^{r_{n}+1}(x+1)^{l_{n}+1} P_{N-2^{n}}^{\left(\alpha+r_{n}+1, \beta+l_{n}+1\right)}(x)\right| \\
& \cdot \int_{l} \frac{|f(z)|}{\left|A_{N+n-2}(z)\right| \cdot|(z-x)|}|d z| .
\end{aligned}
$$

By noticing that the limiting distribution $\mu(x)$ of the zeros of the Jacobi polynomial as $N \rightarrow \infty$ is

$$
\mu(x)=\frac{1}{\pi} \int_{-1}^{x}\left(1-t^{2}\right)^{-1 / 2} d t
$$

and following the procedure in [11], we find from (3.10),

$$
\begin{aligned}
\left|R_{N}[f ; x]\right|=O( & \frac{1}{\gamma_{N-1}} \max _{-1 \leqq x \leqq 1}\left|(x-1)^{r_{n}+1}(x+1)^{l_{n}+1} P_{N-2^{n}}^{\left(\alpha+r^{+}+1, \beta+l_{n}+1\right)}(x)\right| \\
& \left.\cdot e^{+N c} \cdot \max _{z \in l}|f(z)|\right),
\end{aligned}
$$

where $l$ is taken as a contour

$$
l=\left\{z: \ln \left(2 /\left|z+\sqrt{z^{2}-1}\right|\right)=c\right\},
$$

which encloses $[-1,1]$ in its interior.

From $[9$, pp. 63,167$]$ we have

$$
\begin{aligned}
& \max _{-1 \leqq x \leqq 1}\left|(x-1)^{r_{n}+1}(x+1)^{l_{n}+1} P_{N-2^{n}}^{\left(\alpha+r_{n}+1, \beta+l_{n}+1\right)}(x)\right| \\
& \quad=O\left(N^{\max \left\{-1 / 2, \alpha-\left(r_{n}+1\right), \beta-\left(l_{n}+1\right)\right\}}\right)
\end{aligned}
$$

and

$$
\begin{aligned}
\gamma_{N-2} & =2^{-(N-2)}\left(\begin{array}{c}
2 N+\alpha+\beta+r_{n}+l_{n}-2 \\
N-2
\end{array}\right) \\
& =O\left(2^{N} N^{-1 / 2}\right)
\end{aligned}
$$

as $N \rightarrow \infty$. Then we have from (3.11)-(3.14)

$$
\left|R_{N}[f ; x]\right|=O\left(\phi(N) e^{(+N c-N \ln 2)} \max _{z \in l}|f(z)|\right),
$$

where $\phi(N)$ is a certain polynomial in $N$. 
Now let us estimate $\left|R_{N}[f ; x]\right|$ for our problem (3.1)-(3.3).

It is known [8] that the corresponding eigenproblem of (3.1) and (3.3)

$$
\begin{aligned}
& \frac{d^{3} u}{d x^{3}}=\lambda^{3} u \quad \text { in }(-1,1), \\
& u(+1)=0 \text {, } \\
& u^{(\nu)}(-1)=0, \quad 0 \leqq \nu \leqq 1
\end{aligned}
$$

has the negative eigenvalues $\lambda_{k}^{3}(k=1,2, \cdots)$, which satisfy the equation

$$
e^{3 \lambda_{k}}=2 \sin \left(\sqrt{3} \lambda_{k}+\frac{\pi}{6}\right)
$$

This equation has asymptotic solution

$$
\lambda_{k} \sim-\left(k+\frac{1}{6}\right) \frac{\pi}{\sqrt{3}}
$$

and the corresponding eigenfunction is given by

$$
A e^{\lambda_{k} x}+B e^{-\lambda_{k} x / 2} \cos \frac{\sqrt{3} \lambda_{k} x}{2}+C e^{-\lambda_{k} x / 2} \sin \frac{\sqrt{3} \lambda_{k} x}{2},
$$

where $A, B$, and $C$ are constants such that the eigenfunction satisfies the boundary conditions in (3.16).

Then from (3.15) and (3.19) we obtain that

$$
\mid R_{N}\left[e^{\lambda_{k} x} ; x\right]=O\left(\phi(N) \exp \left\{-N x_{0}\left[-\gamma_{k}+\frac{\ln \left(x_{0}+\sqrt{x_{0}^{2}-1}\right)}{x_{0}}\right]\right\}\right),
$$

and

$$
\begin{aligned}
& \left|R_{N}\left[\exp \left\{-\frac{\lambda_{k} x}{2}+i \frac{\sqrt{3} \lambda_{k} x}{2}\right\} ; x\right]\right| \\
& \quad=O\left(\phi(N) \exp \left\{-N\left(x_{0}+\sqrt{3} y_{0}\right)\left[-\frac{\gamma_{k}}{2}+\frac{\ln \left(x_{0}+\sqrt{x_{0}^{2}-1}\right)}{x_{0}+\sqrt{3} y_{0}}\right]\right\}\right),
\end{aligned}
$$

where $x_{0}>1$ and $y_{0}>0$ are the intersection points of the contour (3.12) with the positive half real axis and the positive half imaginary axis, respectively, and the relation between $x_{0}$ and $y_{0}$ is given by

$$
x_{0}+\sqrt{x_{0}^{2}-1}=y_{0}+\sqrt{y_{0}^{2}+1} .
$$

In (3.20) and (3.21) we have used the symbol

$$
\gamma_{k}=\frac{\left|\lambda_{k}\right|}{N}\left(\approx \frac{k}{N} \frac{\pi}{\sqrt{3}}\right)
$$

Noticing that the function $1 / x_{0} \ln \left(x_{0}+\sqrt{x_{0}^{2}-1}\right)$ attains its maximum $(\approx 0.662743)$ at $x_{0} \approx 1.81003$ and the function $\ln \left(x_{0}+\sqrt{x_{0}^{2}-1}\right) /\left(x_{0}+\sqrt{3} y_{0}\right)$ attains its maximum $(\approx 0.280327)$ at $x_{0} \approx 1.45188$, then we have from $(3.20)$ and $(3.21)$ that $R_{N}\left[e^{\lambda_{k} x} ; x\right] \rightarrow 0$ and $R_{N}\left[\exp \left[-\left(\lambda_{k} x / 2\right)+i\left(\sqrt{3} \lambda_{k} x / 2\right)\right] ; x\right] \rightarrow 0$ as $N \rightarrow \infty$ if $\gamma_{k}<0.560654$. Thus we have proved the following theorem. 
THEOREM 3.1. A sufficient condition for convergence in the sense of

$$
\max _{-1 \leqq x \leqq 1}\left|f_{k}(x)-P_{N}(x)\right| \rightarrow 0 \quad \text { as } N \rightarrow \infty
$$

is

$$
\frac{k}{N} \lesssim 0.309105 \quad\left(\approx \frac{3}{10}\right),
$$

where $f_{k}(x)$ is the kth eigenfunction given by (3.19) of eigenproblem (3.16) and $P_{N}(x)$ is its interpolation polynomial defined by (2.3).

A related condition was obtained in [8] for Chebyshev collocation using a different approach.

3.2. Differentiation matrices and numerical observations. In this section we continue to observe the pseudospectral approximation to eigenvalues of (3.16).

By using (3.4) and (3.5) and noticing that $h_{j}(x)$ can be rewritten as

$$
h_{j}(x)=\frac{1+x}{1+x_{j}} l_{j}(x), \quad 2 \leqq j \leqq N-1,
$$

where $l_{j}(x)$ is the Lagrange interpolating polynomial

$$
l_{j}(x)=\prod_{\substack{k=1 \\ k \neq j}}^{N} \frac{x-x_{k}}{x_{j}-x_{k}},
$$

it is not difficult to obtain third-order differentiation matrices as follows:

$$
D_{N}^{(3)}=\left(d_{k j}^{(3)}\right)_{k=2, \cdots, N-1, j=2, \cdots, N-1}
$$

with

$$
d_{k j}^{(3)}=\frac{1}{1+x_{j}}\left[\left(1+x_{k}\right) q_{k, j}^{(3)}+3 q_{k, j}^{(2)}\right], \quad k, j=2, \cdots, N-1
$$

where

$$
\begin{aligned}
& q_{k, j}^{(3)}= \begin{cases}3 q_{k j}\left[\left(q_{k k}\right)^{2}-\frac{2 q_{k k}}{\left(x_{k}-x_{j}\right)}+\frac{2}{\left(x_{k}-x_{j}\right)^{2}}-\sum_{i \neq k} \frac{1}{\left(x_{k}-x_{i}\right)^{2}}\right], & j \neq k, \\
\left(q_{k k}\right)^{3}-3 q_{k k} \sum_{i \neq k} \frac{1}{\left(x_{k}-x_{i}\right)^{2}}+2 \sum_{i \neq k} \frac{1}{\left(x_{k}-x_{i}\right)^{3}}, & j=k,\end{cases} \\
& q_{k j}^{(2)}= \begin{cases}2 q_{k j}\left[q_{k k}-\frac{1}{\left(x_{k}-x_{j}\right)}\right], & j \neq k, \\
\left(q_{k k}\right)^{2}-\sum_{i \neq k} \frac{1}{\left(x_{k}-x_{i}\right)^{2}}, & j=k,\end{cases} \\
& q_{k j}= \begin{cases}\frac{a_{k}}{a_{j}\left(x_{k}-x_{j}\right)}, & j \neq k, \\
\sum_{i \neq k} \frac{1}{x_{k}-x_{i}}, & j=k,\end{cases}
\end{aligned}
$$

and

$$
a_{k}=\prod_{\substack{i=1 \\ i \neq k}}^{N}\left(x_{k}-x_{i}\right) .
$$

In (3.28) the sum is over $i$ from 1 to $N$.

Let the eigenvalues of $D_{N}^{(3)}$ be ordered as

$$
\operatorname{Re}\left(\tau_{1}\right) \geqq \operatorname{Re}\left(\tau_{2}\right) \geqq \cdots \geqq \operatorname{Re}\left(\tau_{N-2}\right) .
$$


The numerically computed $\operatorname{Re}\left(\tau_{1}\right)$ is shown in Fig. 3.1 as a function of parameters $\alpha$ and $\beta$ at $N=32$. Recall that in this section we assume that the interior collocation points are the zeros of $P_{N-2}^{(\alpha+1, \beta+2)}(x)$, and that $\alpha$ and $\beta$ assume values in $(-2, \infty)$ and $(-3, \infty)$, respectively. Observe that when $\beta \geq 0$ (excluding $\beta=0$ ) the scheme (3.5) is unstable since $\operatorname{Re}\left(\tau_{1}\right)>0$. Figure 3.2 shows the function $N^{*}(N, \alpha, \beta)$ which is defined as

$$
N^{*}(N, \alpha, \beta)= \begin{cases}\frac{k^{*}}{N-2} & \text { if } \tau_{k} \text { is real, } \tau_{k}<0 \\ & \text { and } \frac{\left|\tau_{k}-\lambda_{k}^{3}\right|}{\left|\lambda_{k}^{3}\right|}<0.01, \quad 1 \leqq k \leqq k^{*}, \\ 0, & \text { otherwise. }\end{cases}
$$

The value of $N^{*}$ gives the proportion of discrete eigenvalues which closely approximate the corresponding eigenvalues of the continuous problem. Note, again, that the approxi-

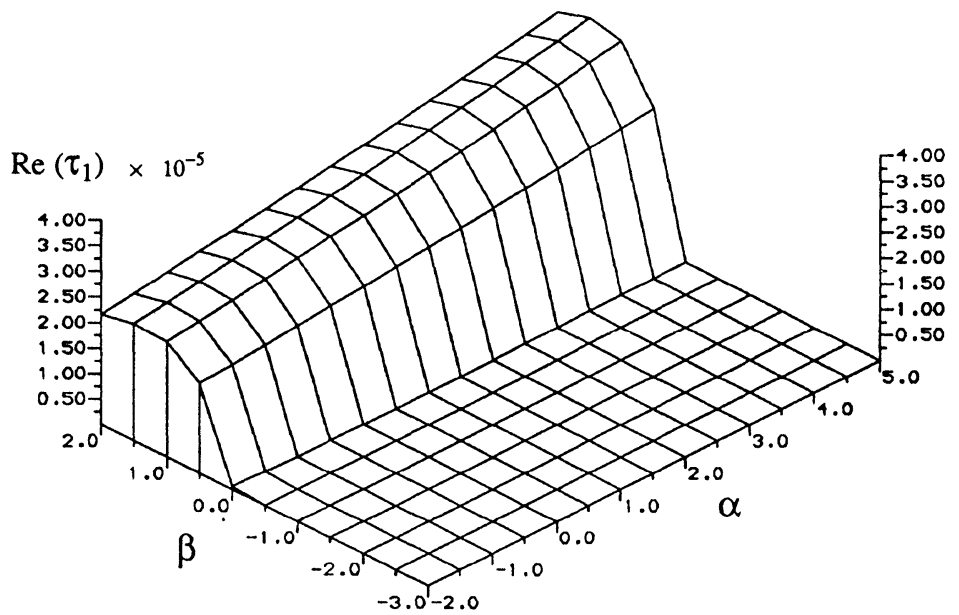

FIG. 3.1. $\operatorname{Re}\left(\tau_{1}\right)$ as a function of $\alpha$ and $\beta$ at $N=32$. $\operatorname{Re}\left(\tau_{1}\right)$ is defined by (3.29).

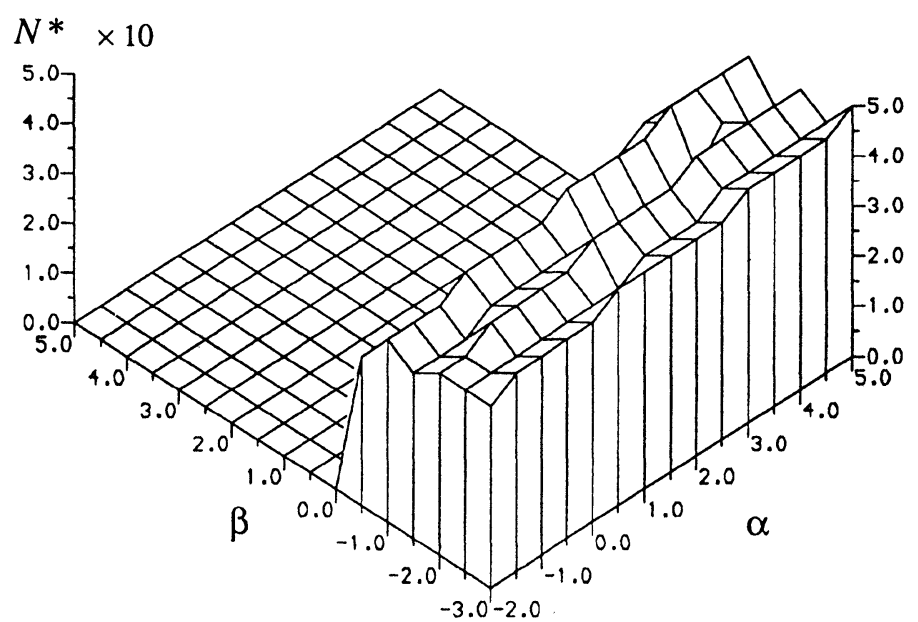

FIG. 3.2. $N^{*}(N, \alpha, \beta)$ as a function of $\alpha$ and $\beta$ at $N=32 . N^{*}(N, \alpha, \beta)$ is defined by (3.30). 
mation process fails if $\beta>0$. Figure 3.3 shows how well the discrete eigenvalue, $\tau_{k}$, approximates the corresponding continuous eigenvalue, $\lambda_{k}^{3}$, for $1 \leqq k \leqq N-2$ and $N=32$. The continuous line and the circles show $\log _{10}\left|\lambda_{k}^{3}\right|$ and $\log _{10}\left|\tau_{k}\right|$, respectively, as functions of $k$. The plots are shown for a selection of parameter sets $(\alpha, \beta)$ in Figs. 3.3(a)-(f). Note, in particular, Figs. 3.3(a)-(c) where the interior collocation points are, respectively, the zeros of $P^{(-1 / 2,-1 / 2)}(x)$ (Lobatto-Chebyshev), $P^{(0,0)}(x)$ (LobattoLegendre), and $P^{(1 / 2,1 / 2)}(x)$ (Lobatto-Chebyshev extrema). In these three cases the

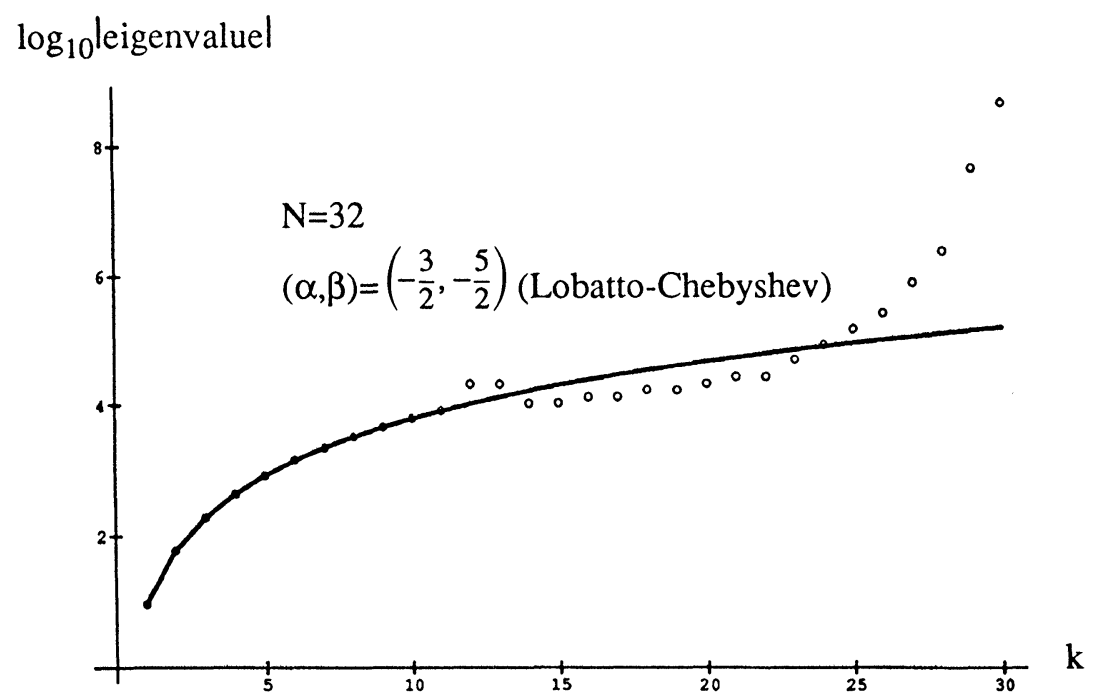

(a)

$\log _{10}$ leigenvaluel

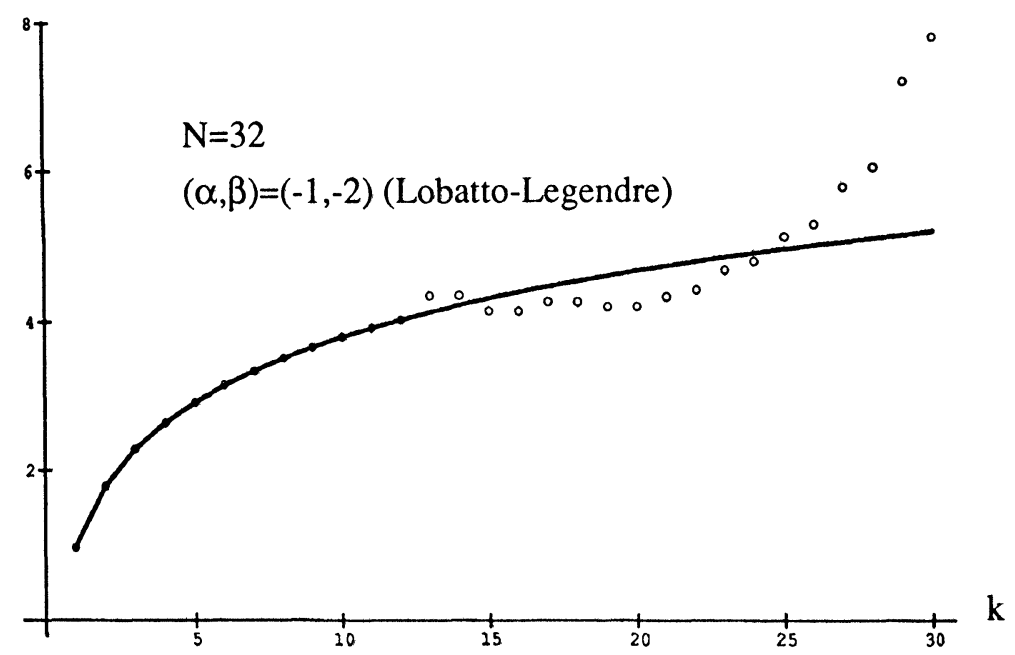

(b)

FIG. 3.3. Comparison of eigenvalues of the continuous problem (3.16) (located on solid line) with those of the discrete problem (located on circles). $\log _{10}\left|\tau_{k}\right|$ and $\log _{10}\left|\lambda_{k}^{3}\right|$ are shown as functions of $k$. 


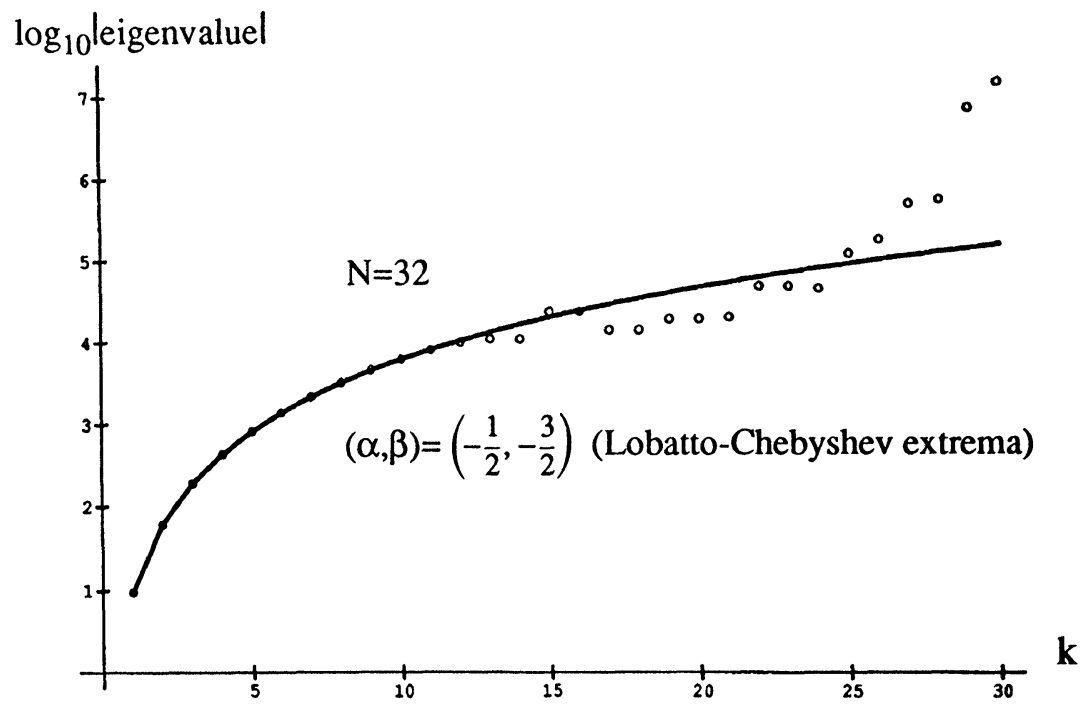

(c)

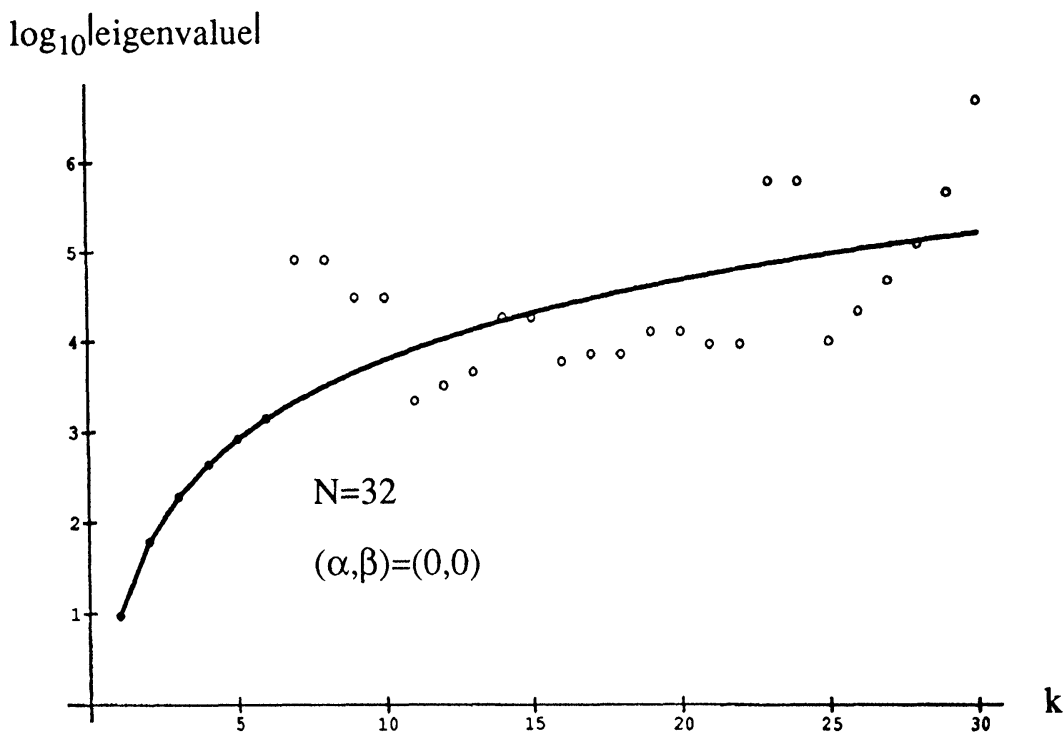

(d)

FIG. 3.3. Continued.

values of $N^{*}$ defined by $(3.30)$ are $0.36667,0.40000$, and 0.36667 , respectively, and the corresponding values of $k^{*}$ are 11,12 , and 11 . The Legendre approximation of the eigenvalues is superior to the two Chebyshev approximations. The best approximation displayed in Fig. 3.3 is that shown in (f) where $(\alpha, \beta)=(3,-1)$ gives $N^{*}=0.43333$ and $k^{*}=13$. It is of interest to note that recent work by Trefethen [10] suggests that since $D_{N}^{(3)}$ is not normal accuracy should be assessed by considering pseudo-eigenvalues rather than exact eigenvalues. This point deserves further examination. 
$\log _{10}$ leigenvaluel

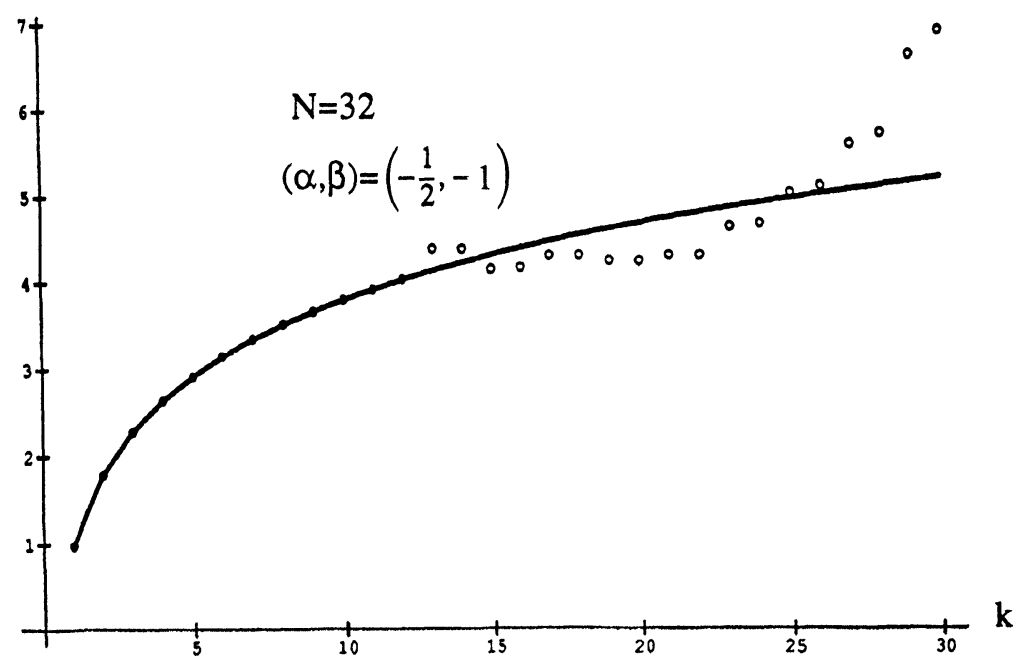

(e)

$\log _{10}$ leigenvaluel

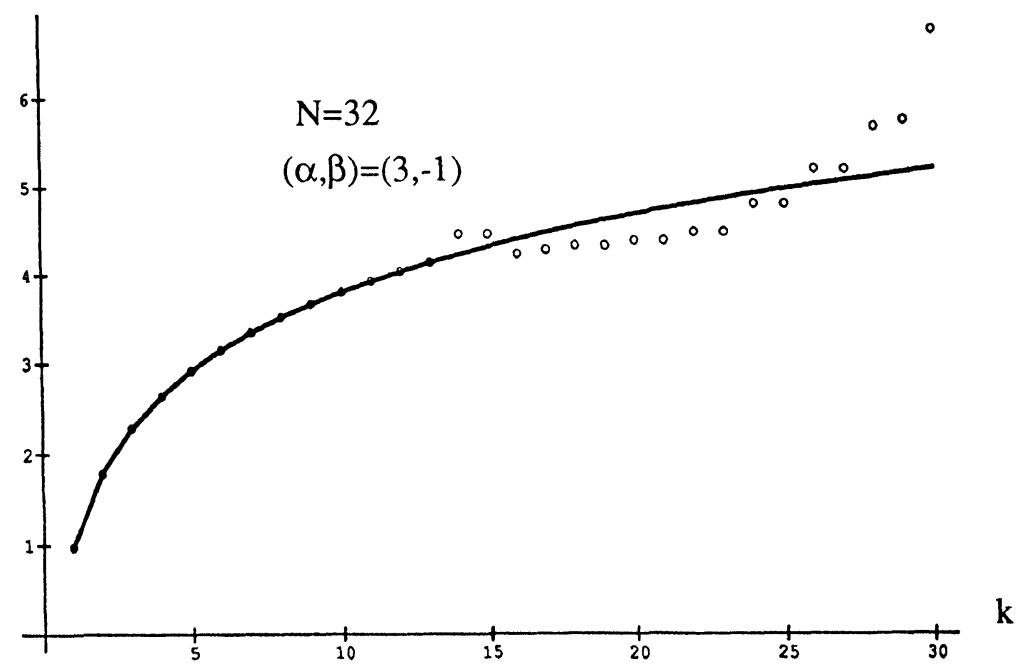

(f)

FIG. 3.3. Continued.

3.3. Convergence. In order to prove convergence we need the following result. LEMMA 3.1. Let $u$ satisfy $u( \pm 1)=0, u^{\prime}(-1)=0$ and have a continuous second-order derivative; then

$$
\int_{-1}^{1} u u_{x x x} d x \leqq 0
$$

Proof. This is trivially established using integration by parts. 
Using this result it is not difficult to prove the stability of the collocation method with $\alpha=0, \beta=0$ for (3.1)-(3.3).

THEOREM 3.2 (Stability). Let $u^{N}$ be the pseudospectral approximation (3.4) with $\alpha=0, \beta=0$ to (3.1)-(3.3). Then

$$
\sum_{k=2}^{N-1}\left[u^{N}\left(x_{k}, t\right)\right]^{2} \omega_{k} \leqq\left[2+O\left(N^{-2}\right)\right] \max _{-1 \leqq x \leqq 1}\left|u_{0}(x)\right|^{2} .
$$

Proof. Multiplying the $k$ th equation of (3.5) by $u^{N}\left(x_{k}, t\right)$ and $\omega_{k}$ and summing over $k$ from 2 to $N-1$, we have

$$
\frac{1}{2} \frac{d}{d t} \sum_{k=2}^{N-1} \omega_{k}\left[u^{N}\left(x_{k}, t\right)\right]^{2}=\sum_{k=2}^{N-1} \omega_{k} u^{N}\left(x_{k}, t\right) \frac{\partial^{3} u^{N}}{\partial x^{3}}\left(x_{k}, t\right) .
$$

Noticing that the degree of polynomial $u^{N}\left(\partial^{3} u^{N} / \partial x^{3}\right)$ is less than $2 N-2$ and that this polynomial satisfies the boundary conditions (3.3), we have from Theorem 2.1 (here $n=3$ )

$$
\frac{1}{2} \frac{d}{d t} \sum_{k=2}^{N-1} \omega_{k}\left[u^{N}\left(x_{k}, t\right)\right]^{2}=\int_{-1}^{1} \omega_{\alpha, \beta}(x) u^{N}(x, t) \frac{\partial^{3} u^{N}}{\partial x^{3}}(x, t) d x .
$$

From Lemma 3.1, we get from $\alpha=0, \beta=0$ that

$$
\frac{d}{d t} \sum_{k=2}^{N-1} w_{k}\left[u^{N}\left(x_{k}, t\right)\right]^{2} \leqq 0
$$

therefore,

$$
\sum_{k=2}^{N-1} \omega_{k}\left[u^{N}\left(x_{k}, t\right)\right]^{2} \leqq \sum_{k=2}^{N-1} \omega_{k}\left[u_{0}\left(x_{k}\right)\right]^{2} .
$$

Then (3.32) follows from (2.33) in Theorem 2.4.

The next step for showing convergence is to show consistency. In order to do this, we first give some results concerning the expansion of functions in Jacobi polynomials.

Lemma 3.2. The Jacobi polynomial $P_{k}^{(\tilde{\alpha}, \tilde{\beta})}(x)(\tilde{\alpha}>-1, \tilde{\beta}>-1)$ satisfies

$$
\frac{d}{d x}\left\{(1-x)^{\tilde{\alpha}+1}(1+x)^{\tilde{\beta}+1} \frac{d}{d x} P_{k}^{(\tilde{\alpha}, \tilde{\beta})}\right\}=-\mu_{k} P_{k}^{(\tilde{\alpha}, \tilde{\beta})}(1-x)^{\tilde{\alpha}}(1+x)^{\tilde{\beta}}
$$

where

$$
\mu_{k}=k(k+\tilde{\alpha}+\tilde{\beta}+1) .
$$

For the proof we refer the reader to [9, pp. 60-61].

LemMA 3.3. For $\tilde{\alpha}>-1, \tilde{\beta}>-1$, we suppose that $u^{(p)}(x), 0 \leqq p \leqq r$, where $r$ is $a$ nonnegative integer, satisfies

$$
\int_{-1}^{1} \omega_{\tilde{\alpha}, \tilde{\beta}}(x)\left|u^{(p)}(x)\right|^{2} d x<+\infty .
$$

Then the coefficients $\tilde{\alpha}_{k}$ of the expansion of $u(x)$ in Jacobi polynomials

$$
u(x)=\sum_{k=0}^{\infty} \tilde{\alpha}_{k} P_{k}^{(\tilde{\alpha}, \tilde{\beta})}(x)
$$


have the asymptotic property

$$
\tilde{\alpha}_{k}=O\left(k^{-2[(r+1) / 2]}\right) \quad \text { as } k \rightarrow \infty .
$$

Proof. Having noticed that the coefficient $\tilde{\alpha}_{k}$ in the expansion may be expressed in the form

$$
\tilde{\alpha}_{k}=\frac{1}{A_{k}} \int_{-1}^{1} \omega_{\tilde{\alpha}, \tilde{\beta}}(x) u(x) P_{k}^{(\tilde{\alpha}, \tilde{\beta})}(x) d x
$$

where

$$
A_{k}=\int_{-1}^{1} \omega_{\tilde{\alpha}, \tilde{\beta}}(x)\left[P_{k}^{(\tilde{\alpha}, \tilde{\beta})}(x)\right]^{2} d x,
$$

we can easily establish the required result by using Lemma 3.2 and integrating by parts.

Lemma 3.4. Suppose that $u(x)$ satisfies boundary conditions

$$
\begin{array}{ll}
u^{(\nu)}(-1)=0, & 0 \leqq \nu \leqq l_{n}, \\
u^{(\nu)}(+1)=0, & 0 \leqq \nu \leqq r_{n}
\end{array}
$$

and has a continuous derivative of order $r\left(\geqq q \equiv \max \left(r_{n}+1, l_{n}+1\right)\right)$. Furthermore, we suppose that

$$
\int_{-1}^{1} \omega_{\alpha+r_{n}+1, \beta+l_{n}+1}(x)\left|\tilde{u}^{(p)}(x)\right|^{2} d x<+\infty, \quad 0 \leqq p \leqq r-q,
$$

where $\tilde{u}(x)$ is the function defined in Lemma 2.2. Then the coefficients $a_{k}$ in the expansion of $u(x)$ in Jacobi polynomials

$$
u(x)=(1-x)^{r_{n}+1}(1+x)^{l_{n}+1} \sum_{k=0}^{\infty} a_{k} P_{k}^{\left(\alpha+r_{n}+1, \beta+l_{n}+1\right)}(x)
$$

have the following asymptotic property:

$$
a_{k}=O\left(k^{-2[(r+1-q) / 2]}\right) \text { as } k \rightarrow \infty .
$$

Proof. From Lemma 2.2, it is known that $\tilde{u}(x)$ is well defined and $\tilde{u}^{(p)}(x)$, $0 \leqq p \leqq r-q$, exists. Then Lemma 3.3 is applied to $\tilde{u}(x)$ with $\tilde{\alpha}=\alpha+r_{n}+1, \tilde{\beta}=\beta+l_{n}+1$ and the conclusion is obtained.

Note 3.1. In the expansion (3.37), the coefficient $a_{k}$ may be expressed as

$$
a_{k}=\int_{-1}^{1} \omega_{\alpha, \beta}(x) u(x) P_{k}^{\left(\alpha+r_{n}+1, \beta+l_{n}+1\right)}(x) d x / A_{k},
$$

where

$$
A_{k}=\int_{-1}^{1} \omega_{\alpha+r_{n}+1, \beta+l_{n}+1}(x)\left[P_{k}^{\left(\alpha+r_{n}+1, \beta+l_{n}+1\right)}(x)\right]^{2} d x .
$$

Note 3.2. The condition (3.36) is not very strong because we can express $u(x)$ as

$$
u(x)= \begin{cases}\frac{\left(1-\theta_{1}\right)^{l_{n}}}{l_{n} !}(1+x)^{l_{n}+1} u^{\left(l_{n}+1\right)}\left(-1+\theta_{1}(x+1)\right), & x \in[-1,0], \\ \frac{\left(1-\theta_{2}\right)^{r_{n}}}{r_{n} !}(-1+x)^{r_{n}+1} u^{\left(r_{n}+1\right)}\left(+1+\theta_{2}(x-1)\right), & x \in[0,1],\end{cases}
$$


by using Taylor expansion with Cauchy remainder. Here $0<\theta_{1}, \theta_{2}<1$ are constants that depend on $u(x)$. Therefore, we can express $\tilde{u}(x)$ as

$$
\tilde{u}(x)= \begin{cases}\frac{\left(1-\theta_{1}\right)^{l_{n}}}{l_{n} !} \frac{u^{\left(l_{n}+1\right)}\left(-1+\theta_{1}(x+1)\right)}{(1-x)^{r_{n}+1}}, & x \in[-1,0], \\ \frac{\left(1-\theta_{2}\right)^{r_{n}}}{r_{n} !} \frac{u^{\left(r_{n}+1\right)}\left(+1+\theta_{2}(x-1)\right)}{(1+x)^{l_{n}+1}}, & x \in[0,1] .\end{cases}
$$

Then, if $u(x) \in C^{\infty}$ and $u(x)$ satisfies the boundary conditions, (3.36) can be satisfied.

Note 3.3. For the values of $l_{n}$ and $r_{n}$ used in this section, we have

$$
a_{k}=O\left(k^{-2[(r-1) / 2]}\right) \quad \text { as } k \rightarrow \infty .
$$

Now we are ready to show consistency for $\alpha>-2, \beta>-3$. In view of the discussion in $[5$, p. 48$]$ the truncation error is given by

$$
\left\|\left[P^{N} \frac{\partial^{3}}{\partial x^{3}} P^{N}-\frac{\partial^{3}}{\partial x^{3}}\right] u\right\|,
$$

where $u \in C^{\infty}$ is the solution to (3.1)-(3.3), $P_{N}$ is a projection operator defined by

$$
P^{N} f=P_{N+n-3}(x) \text {, with } n=3
$$

(where $P_{N+n-3}$ is defined by (2.3)), and

$$
\|g\|=\left[\sum_{k=2}^{N-2} \omega_{k} g^{2}\left(x_{k}\right)\right]^{1 / 2} .
$$

Note 3.4. In the norm defined by (3.41),

$$
\left\|P^{N} g-g\right\|=0 \text { for any } g(x) \text { on }[-1,1] \text {; }
$$

therefore,

$$
\left\|P^{N}\right\| \leqq 1
$$

Theorem 3.3 (Consistency). Let $u, p^{N}$, and $\|\cdot\|$ be defined as above; then

$$
\left\|\left[P^{N} \frac{\partial^{3}}{\partial x^{3}} P^{N}-\frac{\partial^{3}}{\partial x^{3}}\right] u\right\|=O\left(\frac{1}{N^{r}}\right)
$$

for any positive $r$.

Proof. We can expand $u$ and $u^{N} \equiv P^{N} u(x)$ from Note 3.2 as

$$
u(x)=(1-x)(1+x)^{2} \sum_{i=0}^{\infty} a_{i} P_{i}^{(\alpha+1, \beta+2)}(x)
$$

and

$$
u^{N}(x)=(1-x)(1+x)^{2} \sum_{i=0}^{N-3} \alpha_{i} P_{i}^{(\alpha+1, \beta+2)}(x)
$$

where

$$
\begin{aligned}
& a_{i}=\frac{1}{A_{i}} \int_{-1}^{1} \omega_{\alpha, \beta}(x) u(x) P_{i}^{(\alpha+1, \beta+2)}(x) d x, \\
& \alpha_{i}=\frac{1}{A_{i}} \int_{-1}^{1} \omega_{\alpha, \beta}(x) u^{N}(x) P_{i}^{(\alpha+1, \beta+2)}(x) d x .
\end{aligned}
$$


Then for any positive $r$,

$$
a_{i}=O\left(i^{-r}\right) \quad \text { as } i \rightarrow \infty
$$

from Lemma 3.4 and Note 3.2 , due to $u(x) \in C^{\infty}$.

Using Theorem 2.1 and the orthogonality of Jacobi polynomials, we have

$$
\begin{aligned}
\alpha_{i}= & \frac{1}{A_{i}} \sum_{k=2}^{N-1} \omega_{k} u\left(x_{k}, t\right) P_{i}^{(\alpha+1, \beta+2)}\left(x_{k}\right) \\
= & a_{i}+\frac{1}{A_{i}} \sum_{k=2}^{N-1} \omega_{k} \sum_{j=2 N-4-i}^{\infty}\left(1-x_{k}\right)\left(1+x_{k}\right)^{2} \\
& \cdot a_{j} P_{j}^{(\alpha+1, \beta+2)}\left(x_{k}\right) P_{i}^{(\alpha+1, \beta+2)}\left(x_{k}\right) ;
\end{aligned}
$$

therefore,

$$
\begin{aligned}
\left|\alpha_{i}-a_{i}\right| & \leqq \frac{O(1)}{A_{i}} \max _{2 \leqq k \leqq N-1}\left|\sum_{j=2 N-4-i}^{\infty} a_{j} P_{j}^{(\alpha+1, \beta+2)}\left(x_{k}\right) P_{i}^{(\alpha+1, \beta+2)}\left(x_{k}\right)\right| \\
& =O\left(N^{-r}\right)
\end{aligned}
$$

for any positive $r$ from Theorem 2.4 and (3.47). Then

$$
\begin{aligned}
& \left\|\left[P^{N} \frac{\partial^{3}}{\partial x^{3}} P^{N}-\frac{\partial^{3}}{\partial x^{3}}\right] u\right\| \\
& \leqq\left\|\left[P^{N} \frac{\partial^{3}}{\partial x^{3}} P^{N}-P^{N} \frac{\partial^{3}}{\partial x^{3}}\right] u\right\|+\left\|P^{N} \frac{\partial^{3}}{\partial x^{3}} u-\frac{\partial^{3}}{\partial x^{3}} u\right\| \\
& \leqq\left\|P^{N}\right\| \cdot\left\|\frac{\partial^{3}}{\partial x^{3}} P^{N} u-\frac{\partial^{3}}{\partial x^{3}} u\right\|+\left\|P^{N} \frac{\partial^{3}}{\partial x^{3}} u-\frac{\partial^{3}}{\partial x^{3}} u\right\| \\
& \leqq\left\|\sum_{i=0}^{N-3}\left(\alpha_{i}-a_{i}\right) \frac{d^{3}}{d x^{3}}\left[(1-x)(1+x)^{2} P_{i}^{(\alpha+1, \beta+2)}(x)\right]\right\| \\
& +\left\|\sum_{i=N-2}^{\infty} a_{i} \frac{d^{3}}{d x^{3}}\left[(1-x)(1+x)^{2} P_{i}^{(\alpha+1, \beta+2)}(x)\right]\right\| \\
& \leqq O\left(N^{-r}\right)
\end{aligned}
$$

for any positive $r$, from (3.47), (3.48), Theorem 2.4, and the asymptotic properties of the zeros of Jacobi polynomials and of the Jacobi polynomials themselves.

Thus, we have proved that the solution to (3.5) and (3.6) approximates the solution to (3.1)-(3.3) if the interior collocation points are chosen to be the zeros of $P_{N-2}^{(1,2)}(x)$. Convergence is likely to hold for a range of $(\alpha, \beta)$ values but we have established stability via Theorem 3.2 only for $\alpha=\beta=0$. Consistency, on the other hand, may readily be established for general $n$ with $r_{n}$ and $l_{n}$ satisfying (2.2) and $\alpha$ and $\beta$ satisfying (2.9).

4. Numerical results. In this section we give some numerical results on the pseudospectral solution of the model third-order boundary value problem

$$
\begin{gathered}
\frac{d^{3} u}{d x^{3}}=f \quad \text { on }[-1,1], \\
u( \pm 1)=0, \quad u^{\prime}(-1)=0,
\end{gathered}
$$


where $f(x)$ is chosen such that the exact solution of $(4.1)$ is $u(x)=\left(1-x^{2}\right)(1+x) e^{K x}$. Here $K$ is a real parameter that can be freely prescribed. Large negative $K$ gives an exact solution with a steep gradient near $x=-1$ and large positive $K$ gives a steep gradient near $x=+1$.

Tables 4.1(a)-(d) give the error in the $\|\cdot\|_{\infty}$ and $\|\cdot\|_{2}$ norms at increasing values of $N$ and at a range of parameter sets $(\alpha, \beta)$. Results are given in Tables 4.1(a)-(d) for $K=-10,-1,+5$, and +10 , respectively. Note that high accuracy and rapid convergence are achieved as soon as $N$ is large enough to give sufficient resolution. Machine accuracy is obtained at $N=16$ when $K=-1$ and at $N=32$ when $K=5$; at $|K|=10$ the required value of $N$ exceeds 32 . Note that the error pattern at $K=10$ differs insignificantly from that at $K=-10$. The parameter set $(\alpha, \beta)=(3,-1)$ does not perform better on the boundary value problem than the other sets, despite the

\section{TABLE 4.1}

(a)

Error in solving (4.1) with exact solution $u(x)=\left(1-x^{2}\right)(1+x) \exp (-10 x)$.

\begin{tabular}{rllllllllll}
\hline $\begin{array}{l}\alpha=-\frac{3}{2} \\
N\end{array}$ & $\begin{array}{l}\beta=-\frac{5}{2} \\
\|e\|_{\infty}\end{array}$ & $\begin{array}{l}\alpha=-1 \\
\|e\|_{\infty}\end{array}$ & $\begin{array}{l}\beta=-2 \\
\|e\|_{2}\end{array}$ & $\begin{array}{l}\alpha=-\frac{1}{2} \\
\|e\|_{\infty}\end{array}$ & $\begin{array}{l}\beta=-\frac{3}{2} \\
\|e\|_{2}\end{array}$ & $\begin{array}{l}\alpha=0 \\
\|e\|_{\infty}\end{array}$ & $\begin{array}{l}\beta=0 \\
\|e\|_{2}\end{array}$ & $\begin{array}{l}\alpha=3 \\
\|e\|_{\infty}\end{array}$ & $\begin{array}{l}\beta=-1 \\
\|e\|_{2}\end{array}$ \\
\hline 4 & $5.04(3)$ & $5.24(3)$ & $4.90(2)$ & $5.63(2)$ & $6.26(2)$ & $6.70(2)$ & $5.76(2)$ & $7.42(2)$ & $2.33(3)$ & $2.49(3)$ \\
8 & $1.15(3)$ & $1.74(3)$ & $1.43(3)$ & $2.31(3)$ & $5.40(2)$ & $8.60(2)$ & $2.68(3)$ & $4.63(3)$ & $3.75(2)$ & $5.48(2)$ \\
16 & 0.644 & 1.12 & 0.298 & 0.661 & 0.399 & 0.714 & 7.15 & 17.4 & 4.87 & 9.17 \\
32 & $1.46(-11)$ & $4.14(-11)$ & $1.31(-11)$ & $3.35(-11)$ & $8.60(-12)$ & $2.22(-11)$ & $5.40(-10)$ & $1.86(-9)$ & $5.22(-10)$ & $1.41(-9)$
\end{tabular}

(b)

Error in solving (4.1) with exact solution $u(x)=\left(1-x^{2}\right)(1+x) \exp (-x)$.

\begin{tabular}{|c|c|c|c|c|c|c|c|c|c|c|}
\hline$N$ & $\begin{array}{l}\alpha=-\frac{3}{2} \\
\|e\|_{\infty}\end{array}$ & $\begin{array}{l}\beta=-\frac{5}{2} \\
\|e\|_{2}\end{array}$ & $\begin{array}{l}\alpha=-1 \\
\|e\|_{\infty}\end{array}$ & $\begin{array}{l}\beta=-2 \\
\|e\|_{2}\end{array}$ & $\begin{array}{l}\alpha=-\frac{1}{2} \\
\|e\|_{\infty}\end{array}$ & $\begin{array}{l}\beta=-\frac{3}{2} \\
\|e\|_{2}\end{array}$ & $\begin{array}{l}\alpha=0 \\
\|e\|_{\infty}\end{array}$ & $\begin{array}{l}\beta=0 \\
\|e\|_{2}\end{array}$ & $\begin{array}{l}\alpha=3 \\
\|e\|_{\infty}\end{array}$ & $\begin{array}{l}\beta=-1 \\
\|e\|_{2}\end{array}$ \\
\hline 4 & 0.999 & 1.00 & 0.505 & 0.505 & 0.165 & 0.215 & 0.640 & 0.790 & $9.02(-2)$ & $9.82(-2)$ \\
\hline 8 & $1.65(-4)$ & $1.77(-4)$ & $5.68(-5)$ & $8.29(-5)$ & $4.56(-5)$ & $5.92(-5)$ & $2.58(-4)$ & $4.30(-4)$ & $1.72(-4)$ & $2.22(-4)$ \\
\hline 16 & $3.73(-15)$ & $5.85(-15)$ & $1.86(-15)$ & $3.03(-15)$ & $9.85(-16)$ & $1.99(-15)$ & $2.17(-14)$ & $5.24(-14)$ & $2.20(-14)$ & $4.45(-14)$ \\
\hline
\end{tabular}

(c)

Error in solving (4.1) with exact solution $u(x)=\left(1-x^{2}\right)(1+x) \exp (5 x)$.

\begin{tabular}{rllllllllll}
\hline $\begin{array}{l}\alpha=-\frac{3}{2} \\
N\end{array}$ & $\begin{array}{l}\beta=-\frac{5}{2} \\
\|e\|_{\infty}\end{array}$ & $\begin{array}{l}\alpha=-1 \\
\|e\|_{\infty}\end{array}$ & $\begin{array}{l}\beta=-2 \\
\|e\|_{2}\end{array}$ & $\begin{array}{l}\alpha=-\frac{1}{2} \\
\|e\|_{\infty}\end{array}$ & $\begin{array}{l}\beta=-\frac{3}{2} \\
\|e\|_{2}\end{array}$ & $\begin{array}{l}\alpha=0 \\
\|e\|_{\infty}\end{array}$ & $\begin{array}{l}\beta=0 \\
\|e\|_{2}\end{array}$ & $\begin{array}{l}\alpha=3 \\
\|e\|_{\infty}\end{array}$ & $\begin{array}{l}\beta=-1 \\
\|e\|_{2}\end{array}$ \\
\hline 4 & $2.69(2)$ & $2.70(2)$ & $3.57(1)$ & $3.61(1)$ & $1.80(1)$ & $1.81(1)$ & $1.13(1)$ & $1.43(1)$ & $1.84(1)$ & $1.85(1)$ \\
8 & 7.03 & $1.11(1)$ & 4.33 & 5.72 & 2.04 & 3.10 & 8.91 & $1.43(1)$ & 9.48 & $1.18(1)$ \\
16 & $2.73(-5)$ & $5.64(-5)$ & $1.65(-5)$ & $3.66(-5)$ & $1.39(-5)$ & $2.61(-5)$ & $2.13(-4)$ & $5.10(-4)$ & $2.13(-4)$ & $3.99(-4)$ \\
32 & $6.99(-14)$ & $1.87(-13)$ & $1.02(-13)$ & $3.11(-13)$ & $1.34(-13)$ & $3.89(-13)$ & $4.76(-14)$ & $1.43(-13)$ & $7.99(-14)$ & $2.47(-13)$ \\
\hline
\end{tabular}

(d)

Error in solving (4.1) with exact solution $u(x)=\left(1-x^{2}\right)(1+x) \exp (10 x)$.

\begin{tabular}{rllllllllll}
\hline $\begin{array}{l}\alpha=-\frac{3}{2} \\
N\end{array}$ & $\begin{array}{l}\beta=-\frac{5}{2} \\
\|e\|_{\infty}\end{array}$ & $\begin{array}{l}\alpha=-1 \\
\|e\|_{\infty}\end{array}$ & $\begin{array}{l}\beta=-2 \\
\|e\|_{2}\end{array}$ & $\begin{array}{l}\alpha=-\frac{1}{2} \\
\|e\|_{\infty}\end{array}$ & $\begin{array}{l}\beta=-\frac{3}{2} \\
\|e\|_{2}\end{array}$ & $\begin{array}{l}\alpha=0 \\
\|e\|_{\infty}\end{array}$ & $\begin{array}{l}\beta=0 \\
\|e\|_{2}\end{array}$ & $\begin{array}{l}\alpha=3 \\
\|e\|_{\infty}\end{array}$ & $\begin{array}{l}\beta=-1 \\
\|e\|_{2}\end{array}$ \\
\hline 4 & $7.46(3)$ & $7.48(3)$ & $1.27(4)$ & $1.27(4)$ & $7.93(3)$ & $7.98(3)$ & $5.15(3)$ & $5.27(3)$ & $1.30(2)$ & $1.30(2)$ \\
8 & $7.75(3)$ & $9.05(3)$ & $2.91(3)$ & $3.49(3)$ & $1.40(3)$ & $1.83(3)$ & $3.36(3)$ & $5.27(3)$ & $4.35(3)$ & $5.38(3)$ \\
16 & 1.87 & 3.77 & 1.06 & 2.36 & 0.769 & 1.59 & $1.11(1)$ & $2.65(1)$ & $1.16(1)$ & $2.14(1)$ \\
32 & $2.65(-11)$ & $7.92(-11)$ & $2.13(-11)$ & $5.70(-11)$ & $1.74(-11)$ & $4.43(-11)$ & $7.11(-10)$ & $2.44(-9)$ & $7.36(-10)$ & $1.97(-9)$ \\
\hline
\end{tabular}


marginally better performance in approximating the eigenvalues of the differential operator. As stated at the end of $\S 3.2$, it is possible that accuracy assessment based on exact eigenvalues might be misleading [10].

5. Comments. We have considered pseudospectral methods for global polynomial approximation of functions and of solutions to differential equations. Generalized quadrature rules have been presented which identify interior collocation points for the pseudospectral solution of certain model differential equations. In these equations the $n$th derivative of the unknown function is specified in the interior of an interval on the real line and $n$ derivative conditions are specified at the endpoints of the interval. The familiar Gauss-Radau and Gauss-Lobatto nodes for $n=1$ and $n=2$, respectively, are special cases of the general presentation.

The case $n=3$ is examined in more detail. It is shown, inter alia, that convergent pseudospectral methods can be constructed for a model third-order evolutionary equation, and the viability of the suggested choice of collocation points is supported by numerical results for a boundary value problem. It is hoped that the presentation will be useful to those who might be involved in the pseudospectral solution of more general third-order problems in one space dimension. Finally, it is hoped that the results will prove useful for the solution of partial differential equations in more than one space dimension.

\section{REFERENCES}

[1] J. P. BoyD, Chebyshev and Fourier Spectral Methods, Springer-Verlag, New York, 1989.

[2] C. Canuto, M. Y. Hussaini, A. Quarteroni, And T. A. ZAnG, Spectral methods in fluid dynamics, Springer-Verlag, New York, 1987.

[3] A. ERdélyi, Tables of Integral Transforms, Vol. II, McGraw-Hill, New York, 1954.

[4] G. H. GolUb AND J. KAUTSKY, Calculation of Gauss quadrature rules with multiple free and fixed knots, Numer. Math., 41 (1983), pp. 147-163.

[5] D. Gottlieb And S. A. Orszag, Numerical Analysis of Spectral Methods: Theory and Applications, Society for Industrial and Applied Mathematics, CBMS-NSF, Philadelphia, PA, 1977.

[6] V. I. KRYLOV, Approximate Calculation of Integrals, Macmillan, New York, 1962.

[7] A. MALEK AND T. N. PHILliPS, Spectral domain decomposition methods for fourth order problems using generalized Gaussian quadrature rules, Mathematics Department Report ABERNA34, University College of Wales, Aberystwyth, Wales, 1990.

[8] L. S. Mulholland, Ph.D. thesis, University of Strathclyde, Glasgow, Scotland, 1991.

[9] G. SzeGö, Orthogonal polynomials, American Mathematical Society, Providence, RI, 1959.

[10] L. N. Trefethen, Approximation theory and numerical linear algebra, in Algorithms for Approximation II, J. C. Mason and M. G. Cox, eds., Chapman, London, 1990.

[11] J. A. C. WeIDEmAn AND L. N. TREFEthen, The eigenvalues of second-order spectral differentiation matrices, SIAM J. Numer. Anal., 25 (1988), pp. 1279-1298. 